\title{
Incorporating Risk Factors to Identify the Indication of Post-mastectomy Radiotherapy in N1 Breast Cancer Treated with Optimal Systemic Therapy: A Multicenter Analysis in Korea (KROG 14-23)
}

Hae Jin Park, MD'
Kyung Hwan Shin, MD, PhD²
Jin Ho Kim, MD, PhD'
Seung Do Ahn, MD, PhD'
Ja Young Kim, MD
Won Park, MD, PhD
Yong Bae Kim, MD, PhD'
Yeon-Joo Kim, MD
Jin Hee Kim, MD, PhD'
Kyubo Kim, MD, PhD'10
Kyung Ran Park, MD, PhD'11
Hyun Soo Shin, MD, PhD'12
Bae Kwon Jeong, MD, PhD'13
Sun Young Lee, MD, PhD
Suzy Kim, MD, PhD'

${ }^{*}$ A list author's affiliations appears at the end of the paper.

\author{
Correspondence: Kyung Hwan Shin, MD, PhD \\ Department of Radiation Oncology, \\ Seoul National University College of Medicine, \\ 101 Daehak-ro, Jongno-gu, Seoul 03080, Korea \\ Tel: 82-2-2072-2524 \\ Fax: 82-2-765-3317 \\ E-mail: radiat@snu.ac.kr \\ Received August 26, 2016 \\ Accepted October 11, 2016 \\ Published Online October 19, 2016
}

\begin{abstract}
Purpose
In a recent meta-analysis, post-mastectomy radiotherapy (PMRT) reduced any first recurrence (AFR) and improved survival in N1 and N2 patients. We investigated risk factors for AFR in N1 after optimal systemic therapy without PMRT, to define a subgroup of patients who may benefit from PMRT.
\end{abstract}

\section{Materials and Methods}

One thousand three hundred eighty-two pT1-2N1M0 breast cancer patients treated with mastectomy without PMRT between 2005 and 2010 were retrospectively analyzed. Only $0.6 \%$ had no systemic therapy.

\section{Results}

After a median follow-up of 5.9 years, there were 173 AFR (53 loco-regional recurrence [LRR] without distant metastases [DM], 38 LRR with DM, and 82 DM without LRR). The 5-year LRR and AFR rates were $6.1 \%$ and $12.0 \%$, respectively. Multivariate analysis revealed that close resection margin $(p=0.001)$ was the only independent risk factor for LRR. Multivariate analysis for AFR revealed that age $<35$ years $(p=0.025)$, T2 stage $(p=0.004)$, high tumor grade $(p=0.032)$, close resection margin $(p=0.035)$, and triple-negative biological subtype $(p=0.031)$ were independent risk factors. Two or three positive lymph nodes $(p=0.078)$ were considered a marginally significant factor. When stratified by these six factors, the 5-year LRR rates were $3.6 \%$ with $0-1(n=606), 7.5 \%$ with $2-3(n=655)$, and $12.7 \%$ with 4-6 ( $n=93$ ) risk factors. The 5-year AFR rates were $7.1 \%$ with $0-1,15.0 \%$ with $2-3$, and $24.5 \%$ with $4-6$ risk factors.

\section{Conclusion}

Patients with pT1-2N1M0 breast cancer who underwent mastectomy and optimal systemic therapy showed excellent loco-regional control and disease control. The patients with four or more risk factors may benefit from PMRT, and those with two or three risk factors merit consideration of PMRT.

\section{Introduction}

Radiotherapy (RT) has played an important role in the management of breast cancer by eradicating microscopic tumor cells not only for all of the patients treated with breastconserving surgery but for selected patients treated with mastectomy [1-3]. Current guidelines generally recommend
Key words

Breast neoplasms, Post-mastectomy radiotherapy, Risk factors 
recent meta-analysis by the Early Breast Cancer Trialists' Collaborative Group (EBCTCG) [5,6]. The Danish trial showed the substantial benefit of PMRT on loco-regional recurrence (LRR) and overall survival in patients with $\mathrm{N} 1$ and N2 disease. EBCTCG analysis also showed a similar result such that PMRT to the chest wall and regional lymphatics reduced both recurrence and breast cancer mortality in patients with $\mathrm{N} 1$ and N2 disease, even when axillary dissection at least at level II was performed, and systemic therapy was given. Nonetheless, the use of PMRT has been controversial for all T1-T2/N1 patients. One reason is that the absolute risk of any type of recurrence has decreased with modern systemic therapy in recent decades. Another reason is related to breast cancer being not a single entity but biologically distinct diseases [7-9]. The biological subtype can also predict the risk of recurrence and response of treatment as well, although the current guidelines suggest the indication of PMRT based on only tumor and nodal stage. Large randomized trials or meta-analyses including the Danish trial and EBCTCG analysis did not address this issue.

The EBCTCG meta-analysis in 2005 showed that the avoidance of LRR led to a reduction of breast cancer mortality in node-positive patients treated with mastectomy and PMRT [10]. On the other hand, the updated analysis in 2014 showed that the avoidance of any first recurrence (AFR) led to a decrease in breast cancer mortality [5]. In this study, we investigated the risk factors for LRR as well as AFR in N1 patients after optimal systemic therapy but not PMRT, thus defining a subgroup of patients who may or may not benefit from PMRT.

\section{Materials and Methods}

This study was approved by the Korean Radiation Oncology Group (KROG 14-23) and Institutional Review Board of each participating institution. After the approval, we retrospectively reviewed the medical records of breast cancer patients treated with mastectomy without PMRT between 2005 and 2010 at 11 institutions in Korea. Patients with a tumor size $\leq 5 \mathrm{~cm}$ (pT1 and pT2) and 1-3 axillary LN metastases (pN1) were exclusively included in this study. We excluded patients who received neoadjuvant systemic treatment, had distant metastasis at diagnosis, had a history of malignancies other than thyroid cancer, or were diagnosed with bilateral breast cancer. A total of 1,382 breast cancer patients met the eligibility criteria.

Clinico-pathological information of eligible patients was collected; it included the age at diagnosis, menopausal status, tumor histology, tumor size, tumor grade, number of involved and examined LNs, and estrogen receptor (ER), progesterone receptor (PR), human epidermal growth factor receptor 2 (HER2), and Ki-67 status. The positivity of ER, PR, HER2, and Ki-67 was determined by immunohistochemical staining. HER2-positivity was defined as a $3+$ immunohistochemical result or a $2+$ immunohistochemical result confirmed by fluorescence in situ hybridization. The breast cancer subtypes were approximated based on hormone receptor status, HER2 status, and histologic grade. Because Ki-67 status data were incomplete (available only in 757 patients [54.8\%]), we used the histologic grade as a surrogate for Ki-67 based on St. Gallen Expert Consensus [11]. The five surrogate biological subtypes were defined accordingly: luminal A (ER+ or PR+/HER2-/low-intermediate grade), luminal B (ER+ or PR+ / HER2-/ high grade), HER2+ (ER-/ PR-/HER2+), luminal HER2 (ER+ or PR+/HER2+), and triple negative (TN) (ER-/PR-/HER2-).

The primary outcome of interest was AFR, irrespective of LRR or distant metastasis (DM). We defined local recurrence (LR) as tumor recurrence in the ipsilateral chest wall, and regional recurrence (RR) as recurrence in ipsilateral draining LNs (axillary, supraclavicular, or internal mammary LNs). LRR was defined as LR or RR or both. Following the EBCTCG meta-analysis, we used LRR as a first event for statistical analysis. Hereafter, LRR refers to LRR with or without synchronous DM. DM was defined as tumor recurrence outside regions identified as LRR sites. The information on date of death was taken from Korea's national database, in which death by breast cancer was not distinguished from death by other causes. Time to any recurrence or death was measured from the date of mastectomy.

Cumulative incidence function curves for AFR, LRR, and overall mortality were constructed using the Kaplan-Meier method, and comparisons between groups were performed using log-rank tests. Statistical analysis was performed using SPSS ver. 18.0 (SPSS Inc., Chicago, IL). p-values lower than 0.05 were deemed to indicate statistical significance.

\section{Results}

\section{Patient and tumor characteristics}

Table 1 summarizes the demographic and clinico-pathologic parameters, as well as treatment details, of the study cohort. The median age at diagnosis was 48 years (range, 24 to 85 years). All 1,382 patients underwent mastectomy with clear resection margins, and 189 of these patients $(13.7 \%)$ had resection margins less than $2 \mathrm{~mm}$. The most common histology was invasive ductal carcinoma (97.5\%). There were 820 
Table 1. Patient, tumor, and treatment characteristics

\section{Variable}

No. $(\%)(\mathrm{n}=1,382)$

\begin{tabular}{|c|c|}
\hline Age, median (range, yr) & $48(24-85)$ \\
\hline \multicolumn{2}{|l|}{ Menopausal status } \\
\hline Premenopausal & $841(60.9)$ \\
\hline Postmenopausal & $530(38.4)$ \\
\hline Unknown & $11(0.8)$ \\
\hline \multicolumn{2}{|l|}{ T stage } \\
\hline $\mathrm{T} 1$ & $562(40.7)$ \\
\hline $\mathrm{T} 2$ & $820(59.3)$ \\
\hline \multicolumn{2}{|l|}{ Tumor histologic grade } \\
\hline Low-intermediate & $807(58.4)$ \\
\hline High & $547(39.6)$ \\
\hline Unknown & $28(2.0)$ \\
\hline \multicolumn{2}{|l|}{ Resection margin } \\
\hline Negative ( $\geq 2 \mathrm{~mm}$ ) & $1,193(86.3)$ \\
\hline Close $(<2 \mathrm{~mm})$ & $189(13.7)$ \\
\hline \multicolumn{2}{|l|}{ No. of nodes examined } \\
\hline Median & $15(1-64)$ \\
\hline$<10$ & 275 (19.9) \\
\hline$\geq 10$ & $1,107(80.1)$ \\
\hline \multicolumn{2}{|l|}{ No. of positive nodes } \\
\hline 1 & $813(58.8)$ \\
\hline 2 & $371(26.8)$ \\
\hline 3 & $198(14.3)$ \\
\hline \multicolumn{2}{|l|}{ Hormonal receptor } \\
\hline Positive & $1,022(74.0)$ \\
\hline Negative & $360(26.0)$ \\
\hline \multicolumn{2}{|l|}{ HER2 amplification } \\
\hline Positive & $407(29.5)$ \\
\hline Negative & 917 (66.4) \\
\hline Unknown & $58(4.2)$ \\
\hline \multicolumn{2}{|l|}{ Systemic treatment } \\
\hline Endocrine therapy alone & $41(3.0)$ \\
\hline Chemotherapy alone & $328(23.7)$ \\
\hline Both & $1,005(72.7)$ \\
\hline Neither & $8(0.6)$ \\
\hline \multicolumn{2}{|l|}{ Chemotherapy } \\
\hline Taxane-containing & $1,104(79.9)$ \\
\hline Non-taxane containing & $229(16.6)$ \\
\hline Not done & $49(3.5)$ \\
\hline \multicolumn{2}{|l|}{ Trastuzumab } \\
\hline HER2+ and trastuzumab- & $154(11.1)$ \\
\hline HER2+ and trastuzumab+ & $253(18.3)$ \\
\hline \multicolumn{2}{|l|}{ Biological subtype } \\
\hline Luminal A & $554(40.1)$ \\
\hline Luminal B & $187(13.5)$ \\
\hline Luminal HER2 & $209(15.1)$ \\
\hline HER2+ & $198(14.3)$ \\
\hline Triple negative & $157(11.4)$ \\
\hline Unknown & $77(5.6)$ \\
\hline
\end{tabular}

HER2, human epidermal growth factor receptor 2.
Table 2. Patterns of failure

\begin{tabular}{lc} 
Site of recurrence & No. (\%) \\
\hline Local & $39(2.8)$ \\
\hline Regional & $70(5.1)$ \\
$\quad$ Axilla & $54(3.9)$ \\
\hline Internal mammary & $36(2.6)$ \\
\hline Supraclavicular & $47(3.4)$ \\
\hline Loco-regional & $91(6.6)$ \\
Distant & $138(10.0)$ \\
\hline
\end{tabular}

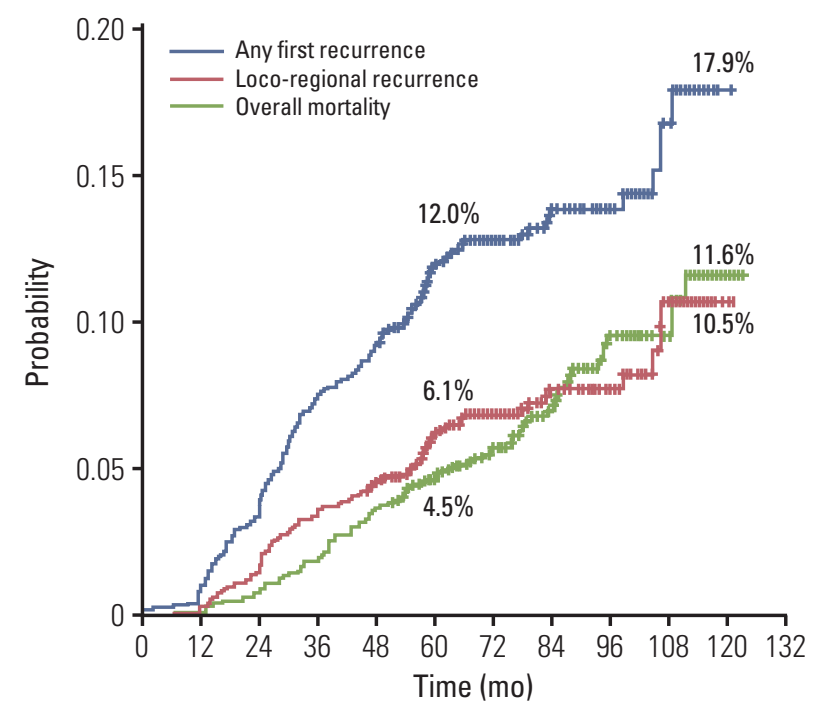

Fig. 1. Cumulative incidence of loco-regional recurrence, any first recurrence, and overall mortality.

T2 tumors (59.3\%), and 547 high-grade tumors (39.6\%). Complete axillary LN dissection was performed in 1,276 patients (92.3\%), and sentinel LN biopsy alone was performed in 103 patients $(7.5 \%)$. The median number of examined LNs was 15 (range, 1 to 64 ), and more than 10 LNs were examined in 1,107 patients $(80.7 \%)$. In all, 813 patients $(58.8 \%)$ had one involved LN, 371 (26.8\%) had two involved LNs, and 198 $(14.3 \%)$ had three involved LNs. There were 1,022 patients $(74.0 \%)$ with positive hormone receptor status, and 14 of them did not receive hormone treatment. On the other hand, 38 of 360 patients with negative hormone receptor status did receive hormone treatment. There were 407 patients $(29.5 \%)$ with positive HER2 expression; $62.1 \%$ of them $(n=253)$ were treated with trastuzumab. In addition, 1,333 (96.5\%) and $1,046(75.7 \%)$ patients received adjuvant chemotherapy $(83 \%$ received a taxane-containing regimen) and hormonal therapy, respectively. Eight patients $(0.6 \%)$ did not receive sys- 


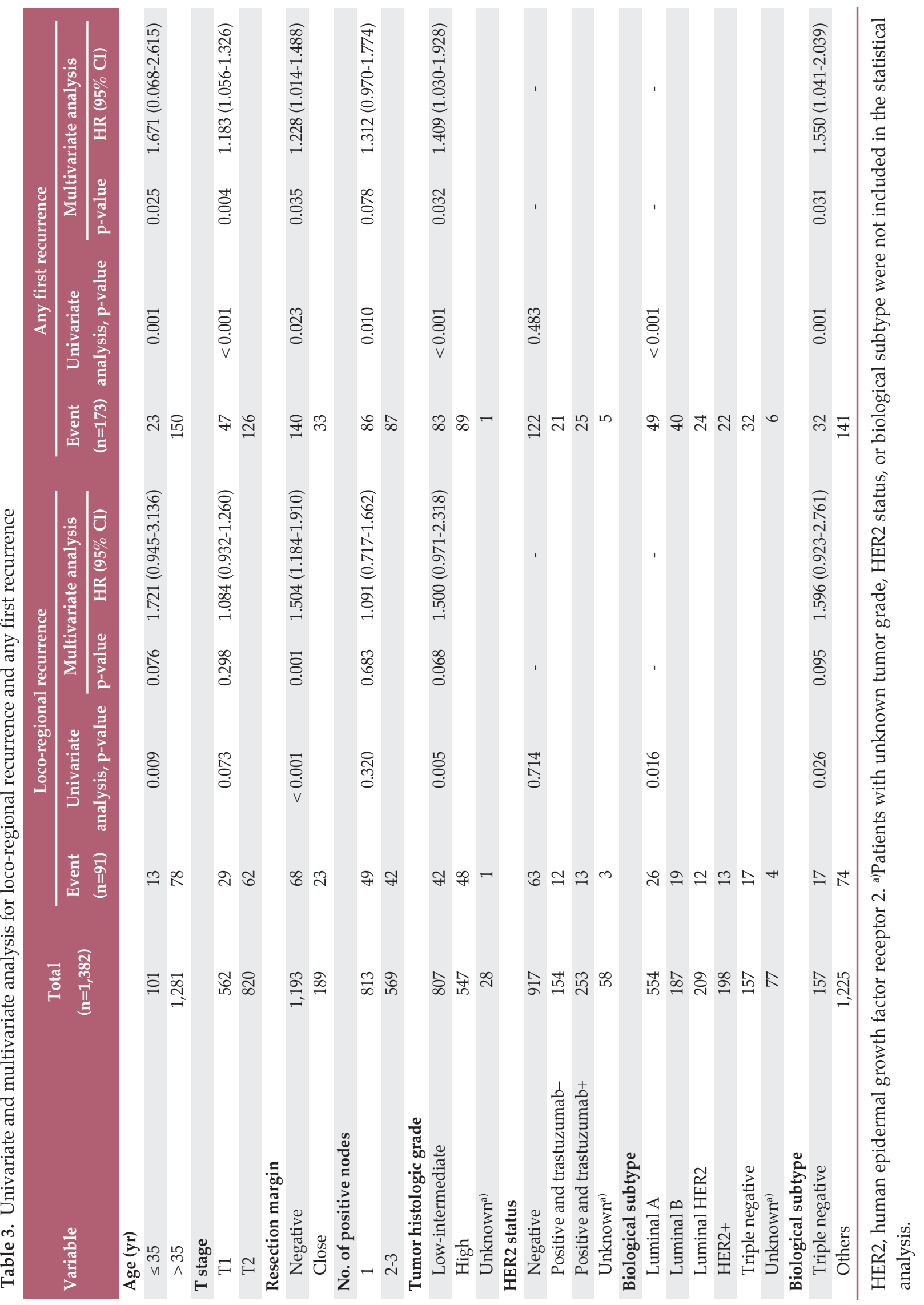

742 CANCER RESEARCH AND TREATMENT 
temic treatment. Adjuvant systemic treatment was delivered at the physician's discretion.

\section{Tumor recurrence and death}

The median follow-up period was 5.9 years (range, 0.6 to 10.4 years). In total, 94 patients $(6.8 \%)$ died, and 79 of them had experienced tumor recurrence before death. Table 2 shows the sites of total recurrence. There were more distant recurrences $(\mathrm{n}=138)$ than LRRs $(\mathrm{n}=91)$. Of 91 LRRs, 17 were synchronous LR and RR. Fifty-seven patients experienced LRR as well as DM. Finally, 81 had isolated DM.

There were 173 AFRs, among which $53(30.6 \%)$ were LRRs without DM, 38 (22.0\%) were LRRs with DM, and 82 (47.4\%) were DMs without LRR. The cumulative incidence curves of LRR, AFR, and overall mortality are illustrated in Fig. 1. The cumulative incidence of AFR and LRR increased steeply within 5 years from the date of mastectomy, and the mortality curve was not particularly steep during the specific time period. The cumulative LRR rates at 5, 7, and 10 years were $6.1 \%, 7.7 \%$, and $10.5 \%$, respectively. Those for AFR at 5, 7, and 10 years were $12.0 \%, 13.8 \%$, and $17.9 \%$, respectively; and those for overall mortality at 5,7 , and 10 years were $4.5 \%$, $6.9 \%$, and $11.6 \%$, respectively.

\section{Effect of biological subtype on LRR and AFR}

When classified into five biological subtypes, luminal A was the most common subtype (40.1\%), and the remaining four subtypes had similar proportions (Table 1). The 5-year AFR rates of luminal A, luminal B, HER2, luminal HER2, and TN were $8.2 \%, 11.7 \%, 10.5 \%, 11.2 \%$, and $18.8 \%$, respectively. And the 10-year AFR rates were 16.7\%, 24.7\%, 13.6\%, 12.6\%, and $25.5 \%$, respectively (S1 Fig. 1A). The 5-year locoregional recurrence-free survival rates of luminal A, luminal B, HER2, luminal HER2, and TN were $4.3 \%, 9.5 \%, 5.9 \%, 5.3 \%$, and $10.4 \%$, respectively. And the 10-year AFR rates were $9.4 \%$, $12.7 \%, 9.2 \%, 6.8 \%$, and $14.6 \%$, respectively (S1 Fig. 1B). In univariate analyses, the TN and luminal B subtypes predicted more LRR and AFR than the luminal A subtype (all, $\mathrm{p}<0.001$ ) (Table 3).

Because the use of trastuzumab for HER2 patients was not covered with Korean national health insurance in the early period of enrollment, 253 of 407 HER2 patients (62.2\%) were treated with trastuzumab. For this reason, we subdivided HER2-positive patients (among the HER2 and luminal HER2 subtypes) into those treated with and without trastuzumab. Thus, we reclassified those patients into five biological subtypes: luminal A, luminal B, HER2- trastuzumab+, HER2trastuzumab-, and TN. The results were similar to those of the former analysis in which the TN and luminal B subtypes had significantly more LRR and AFR than the luminal A sub- type (all, $\mathrm{p}<0.001$ ). There was slightly more LRR and AFR in the HER2 group without trastuzumab treatment than in the HER2 group with the treatment, but this difference was not statistically significant ( $\mathrm{p}=0.674$ and $\mathrm{p}=0.415$, respectively).

\section{Risk factors}

The results of univariate and multivariate analyses of LRR and AFR are presented in Table 3. To facilitate the comparison between groups in multivariate analyses using the Cox proportional hazards model, the biological subtypes were redefined as binary variables: $\mathrm{TN}$ tumors and others $(\mathrm{p}=0.001)$ (Table 3).

Univariate analyses of LRR revealed that age $\leq 35$ years, a close resection margin, and a high tumor grade were significantly associated with a high LRR ( $p=0.009, p<0.001$, and $p=0.005$, respectively). T2 stage was marginally significant with a high LRR ( $\mathrm{p}=0.073$ ). In multivariate analyses of LRR, a close resection margin was the only independent risk factor (hazard ratio [HR], 1.504; $\mathrm{p}=0.001$ ), and age (HR, 1.721; $\mathrm{p}=0.076)$, high tumor grade $(\mathrm{HR}, 1.500 ; \mathrm{p}=0.068)$, and TN biological subtype $(H R, 1.596 ; p=0.095)$ were marginally significant factors for LRR.

Univariate analyses of AFR revealed that age $\leq 35$ years, T2 stage, a close resection margin, two or three positive LNs, positive $\mathrm{HR}$, and high tumor grade were independent risk factors for AFRs $(\mathrm{p}=0.001, \mathrm{p}<0.001, \mathrm{p}=0.023, \mathrm{p}=0.010$, $\mathrm{p}=0.483$, and $\mathrm{p}<0.001$, respectively). In multivariate analyses of AFRs, age $\leq 35$ years (HR, 1.671; $\mathrm{p}=0.025)$, T2 stage (HR, $1.183 ; \mathrm{p}=0.004)$, a close resection margin $(\mathrm{HR}, 1.228 ; \mathrm{p}=0.035)$, a high tumor grade (HR, 1.409; $\mathrm{p}=0.032)$, and the TN biological subtype (HR, 1.550; $\mathrm{p}=0.031$ ) were independent risk factors. Two or three positive LNs (HR, 1.312; $\mathrm{p}=0.078$ ) were a marginally significant factor for AFR.

\section{Risk stratification to identify PMRT indication}

Because the purpose of our study was to identify patients who may benefit from PMRT mainly based on overall recurrence, we utilized risk factors proven to be independent in multivariate analyses for AFRs to define risk groups. The six risk factors were patients' age ( $\leq 35$ years vs. $>35$ years), tumor size (T1 vs. T2), the resection margin status (negative vs. close), the number of metastatic LNs (1 vs. 2-3), tumor grade (low-intermediate vs. high), and biological subtype (TN vs. others). Two patients had no risk factors, and 411, 414, 241, 76, and 17 patents had one, two, three, four, and five risk factors, respectively. No patients had all six risk factors.

The risk of both LRR and AFR increased with increasing number of risk factors. The results stratified by risk group are illustrated in Fig. 2. The 5-year cumulative LRR rates 
A

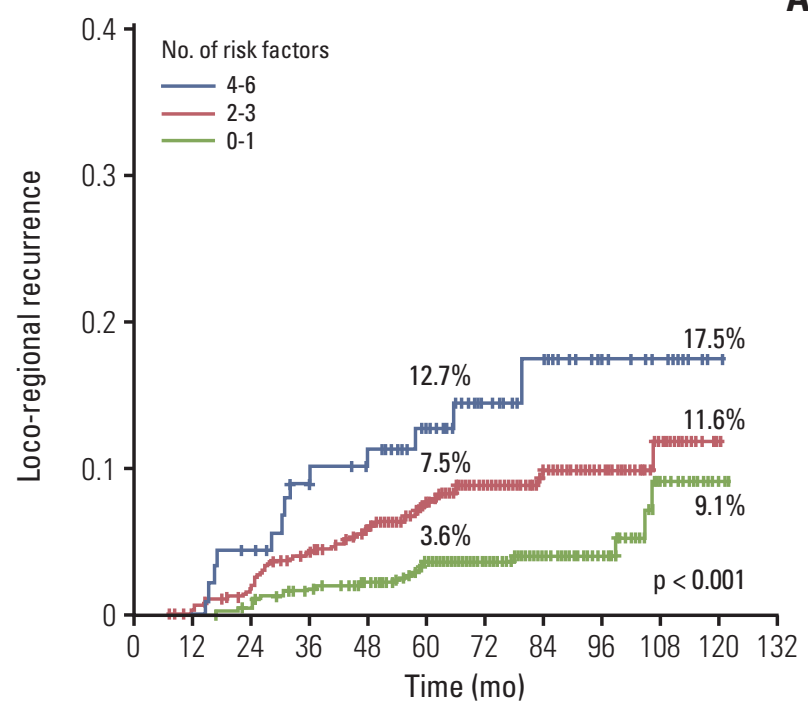

Fig. 2. Increased risk of loco-regional recurrence (A) and any first recurrence (B) with increasing number of risk factors.

were $3.6 \%$ with $0-1$ risk factor $(\mathrm{n}=606), 7.5 \%$ with $2-3$ risk factors $(\mathrm{n}=655)$, and $12.7 \%$ with $4-6$ risk factors $(\mathrm{n}=93)$. The 10-year cumulative LRR rates were $9.1 \%$ with $0-1$ risk factor, $11.6 \%$ with $2-3$ risk factors, and $17.5 \%$ with $4-6$ risk factors (Fig. 2A). The 5-year cumulative AFR rates were $7.1 \%$ with 0-1 risk factor, $15.0 \%$ with $2-3$ risk factors, and $24.5 \%$ with 4-6 risk factors. The 10-year cumulative AFR rates were $12.8 \%$ with $0-1$ risk factor, $22.3 \%$ with $2-3$ risk factors, and $30.5 \%$ with $4-6$ risk factors (Fig. 2B).

\section{Discussion}

PMRT following mastectomy in pT1-2N1 breast cancer patients has not been generally recommended because of the low risk of recurrence. Although the updated EBCTCG metaanalysis proved the benefit of PMRT in N1 and N2 patients who had axillary dissection and systemic therapy [5], the recent consensus from the St. Gallen breast cancer meeting in 2015 did not adopt the routine use of PMRT for all N1 patients, just those with adverse pathology [1]. The present study was designed to identify a subset of N1 patients who may benefit from PMRT.

The entire cohort did not receive PMRT after mastectomy. Our analysis showed that the 10-year AFR and LRR rates were $17.9 \%$ and $10.5 \%$, respectively. These rates are comparable to those of the recent contemporary study by Lai et al. [12], which include almost the same study population with

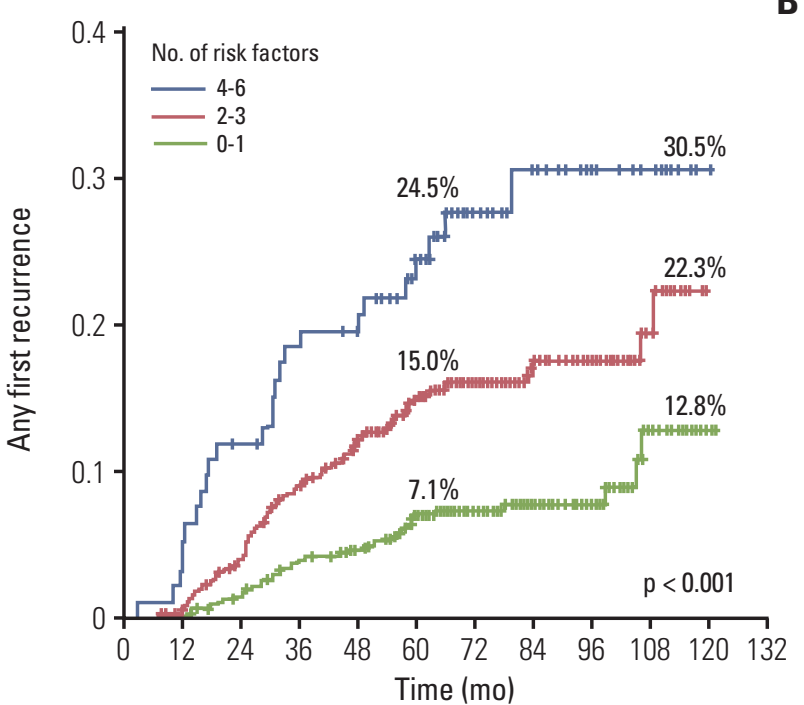


$80 \%$ (1,104 of 1,382 patients) received a taxane-containing chemotherapy, and one-third of the patients received an aromatase inhibitor as endocrine therapy; more than half of the HER2-positive patients received trastuzumab in our cohort. Therefore, it is reasonable that these superior results of our analysis are attributable to modern systemic treatment $[13,14]$; however, further discussion is beyond the scope of this study. The final possible explanation is that our data were immature to exhibit entire recurrence considering the long natural history of breast cancer. The studies included in the EBCTCG meta-analysis were carried out between 1964 and 1986, and the patients were followed up for more than 10 years (median follow-up period, 9.4 years). The patients in our study were treated between 2005 and 2010, and the median follow-up period was 5.9 years.

Although the difference in the LRR and AFR rates between our study and the EBCTCG meta-analysis seems large, the relationship between them is similar. The closest subset to our cohort in the EBCTCG meta-analysis was the N1 patients treated with systemic treatment and without PMRT. In this subset, the 5-year LRR rate was $17.4 \%$ and the 10-year AFR rate was $45.5 \%$, which was 2.6 times higher. In our study, the 5-year LRR rate was $6.1 \%$ and the 10-year AFR rate was $17.9 \%$, which was 2.9 times higher. Considering that PMRT reduced any recurrence by one-third in the EBCTCG metaanalysis, if PMRT were administered, the 10-year overall recurrence would be decreased to $12 \%$. The EBCTCG data also showed that the 10-year AFR rate and 20-year breast cancer mortality rate were similar, and PMRT reduced the 20 -year breast cancer mortality by more than one-fifth. We predict that the 20-year breast cancer mortality would be $18 \%$; if PMRT were administered, this rate would decrease to $14 \%$ in our cohort.

Classification of breast cancer according to biological subtype has proven to be a strong predictor of LRR, DM, and survival [9,15-19]. As mentioned above, the patients in the EBCTCG meta-analysis were diagnosed and treated more than 30 years ago. Thus, it did not address the effects of biological subtypes on the risk of recurrence, which is being utilized for treatment decision making and predicting the prognosis. This is one reason why PMRT could not be recommended for all N1 patients, despite evidence that PMRT does reduce LRR and increase survival. Our study's finding that patients with the luminal A subtype had lower rates of LRR and those with the TN subtype had higher rates of LRR relative to other subtypes is similar to other studies in the setting of mastectomy $[12,16,18,20]$. In the systemic review by Lowery et al. [16], the luminal, HER2-overexpressing, and TN tumor subtypes were defined according to the expression of ER, PR, and HER receptor as determined by immunohistochemical staining. Luminal tumors were less likely to develop LRR than HER2-overexpressing or TN tumors, but there were no differences in LRR between HER2-overexpressing and $\mathrm{TN}$ tumors following mastectomy. Tseng et al. [18] defined five biological subtypes, which were the same as ours, and evaluated LRR after mastectomy and the impact of PMRT by breast cancer subtype. Compared to luminal A patients, TN patients had the highest risk of LRR and the least benefit from PMRT. Patients with HER2 tumors treated with trastuzumab had a low risk of LRR. However, in our study, the use of trastuzumab in HER2 tumors did not affect LRR or AFR.

We defined AFR as the primary endpoint, similar to the EBCTCG meta-analysis. Therefore, to suggest a PMRT indication in N1, we utilized the risk factors by multivariate analyses of AFR to identify a high-risk subgroup. The 10-year AFR rates were as follows: $30.5 \%$ with four or more risk factors present simultaneously (young age, T2 tumors, a close resection margin, high-grade tumors, and the TN subtype); $22.3 \%$ with $2-3$ risk factors, and $12.8 \%$ with $0-1$ risk factor. If the proportional risk reductions were applied to each risk group after adding PMRT, the 10-year AFR rates would decline to the following: $22.8 \%$ with 4 or more risk factors, $16.7 \%$ with $2-3$ risk factors, and $9.6 \%$ with $0-1$ risk factor, respectively. However, in the latter group, the absolute gain of the overall recurrence would be only $3.2 \%$.

This retrospective study had several limitations. First, pathologic findings such as HR/HER2 positivity or tumor grade were not centrally reviewed or reassessed according to common criteria. Criteria discrepancies among participating institutions might hamper an accurate analysis. Second, the follow-up period was relatively short, and our data on patient deaths were all-cause mortality. Therefore, our study could not address survival benefits related to overall recurrence reduction obtained by PMRT in T1-2N1 patients. Despite these limitations, our study had important strengths. This was a large cohort study in which 1,382 T1-2N1 breast cancer patients treated with mastectomy without PMRT were included. About half $(\mathrm{n}=682)$ patients were included in $\mathrm{N} 1$ and no-RT subset of EBCTCG meta-analysis compared to our study cohort. More importantly, because our study adopted current diagnostic and therapeutic strategies, the conclusions drawn by our analyses are more relevant to real practice than those of the EBCTCG meta-analysis.

The United Kingdom Medical Research Council SUPREMO (Selective Use of Postoperative Radiotherapy After Mastectomy) trial, which randomly allocated approximately 1,600 patients with high-risk N0 as well as N1, completed patients accrual [21]. We expect that the result of this trial help clarify the indication of PMRT. 


\section{Conclusion}

In summary, patients with pT1-2N1 breast cancer who underwent mastectomy and optimal systemic therapy showed favorable LRR and overall recurrence without PMRT. However, the concomitant presence of multiple risk factors contributes to higher tumor recurrence. The patients with four or more risk factors may benefit from PMRT, and those with two or three risk factors merit consideration of PMRT. In addition, PMRT may be omitted for patients without risk factors and with only one risk factor because the absolute benefits are small.

\section{Electronic Supplementary Material}

Supplementary materials are available at Cancer Research and Treatment website (http:// www.e-crt.org).

\section{Conflicts of Interest}

Conflict of interest relevant to this article was not reported.

\section{Author Details}

Department of Radiation Oncology, ${ }^{1}$ Hanyang University Hospital, Seoul, ${ }^{2}$ Seoul National University College of Medicine, Seoul, '3Seoul National University Hospital, Seoul, ${ }^{4}$ Asan Medical Center, University of Ulsan College of Medicine, Seoul, ${ }^{5}$ Asan Medical Center, Seoul, ${ }^{6}$ Samsung Medical Center, Sungkyunkwan University School of Medicine, Seoul, ${ }^{7}$ Yonsei Cancer Center, Yonsei University College of Medicine, Seoul, ${ }^{8}$ Proton Therapy Center, National Cancer Center, Goyang, ${ }^{9}$ Dongsan Medical Center, Keimyung University School of Medicine, Daegu, ${ }^{10}$ Ewha Womans University School of Medicine, Seoul, ${ }^{11}$ Ewha Womans University Mokdong Hospital, Seoul, ${ }^{12} \mathrm{CHA}$ Bundang Medical Center, CHA University School of Medicine, Seongnam, ${ }^{13}$ Gyeongsang National University Hospital, Gyeongsang National University School of Medicine, Jinju, ${ }^{14}$ Chonbuk National University Hospital, Jeonju, ${ }^{15}$ Seoul Metropolitan Government Seoul National University Boramae Medical Center, Seoul, Korea

\section{References}

1. Coates AS, Winer EP, Goldhirsch A, Gelber RD, Gnant M, Piccart-Gebhart M, et al. Tailoring therapies: improving the management of early breast cancer: St Gallen International Expert Consensus on the Primary Therapy of Early Breast Cancer 2015. Ann Oncol. 2015;26:1533-46.

2. Gradishar WJ, Anderson BO, Balassanian R, Blair SL, Burstein HJ, Cyr A, et al. Invasive Breast Cancer Version 1.2016, NCCN Clinical Practice Guidelines in Oncology. J Natl Compr Canc Netw. 2016;14:324-54.

3. Kang JK, Kim MS, Jang WI, Seo YS, Kim HJ, Cho CK, et al. The clinical utilization of radiation therapy in Korea between 2009 and 2013. Radiat Oncol J. 2016;34:88-95.

4. Recht A, Edge SB, Solin LJ, Robinson DS, Estabrook A, Fine $\mathrm{RE}$, et al. Postmastectomy radiotherapy: clinical practice guidelines of the American Society of Clinical Oncology. J Clin Oncol. 2001;19:1539-69.

5. EBCTCG (Early Breast Cancer Trialists' Collaborative Group), McGale P, Taylor C, Correa C, Cutter D, Duane F, et al. Effect of radiotherapy after mastectomy and axillary surgery on 10-year recurrence and 20-year breast cancer mortality: metaanalysis of individual patient data for 8135 women in 22 randomised trials. Lancet. 2014;383:2127-35.

6. Overgaard M, Nielsen HM, Overgaard J. Is the benefit of postmastectomy irradiation limited to patients with four or more positive nodes, as recommended in international consensus reports? A subgroup analysis of the DBCG 82 b\&c randomized trials. Radiother Oncol. 2007;82:247-53.

7. Jatoi I, Anderson WF, Jeong JH, Redmond CK. Breast cancer adjuvant therapy: time to consider its time-dependent effects. J Clin Oncol. 2011;29:2301-4.

8. Perou CM, Sorlie T, Eisen MB, van de Rijn M, Jeffrey SS, Rees $\mathrm{CA}$, et al. Molecular portraits of human breast tumours. Nature. 2000;406:747-52.

9. Sorlie T, Perou CM, Tibshirani R, Aas T, Geisler S, Johnsen H, et al. Gene expression patterns of breast carcinomas distinguish tumor subclasses with clinical implications. Proc Natl Acad Sci U S A. 2001;98:10869-74.

10. Early Breast Cancer Trialists' Collaborative Group (EBCTCG). 
Effects of chemotherapy and hormonal therapy for early breast cancer on recurrence and 15-year survival: an overview of the randomised trials. Lancet. 2005;365:1687-717.

11. Goldhirsch A, Wood WC, Coates AS, Gelber RD, Thurlimann B, Senn HJ, et al. Strategies for subtypes: dealing with the diversity of breast cancer: highlights of the St. Gallen International Expert Consensus on the Primary Therapy of Early Breast Cancer 2011. Ann Oncol. 2011;22:1736-47.

12. Lai SF, Chen YH, Kuo WH, Lien HC, Wang MY, Lu YS, et al. Locoregional recurrence risk for postmastectomy breast cancer patients With T1-2 and one to three positive lymph nodes receiving modern systemic treatment without radiotherapy. Ann Surg Oncol. 2016;23:3860-9.

13. Early Breast Cancer Trialists' Collaborative Group (EBCTCG), Davies C, Godwin J, Gray R, Clarke M, Cutter D, et al. Relevance of breast cancer hormone receptors and other factors to the efficacy of adjuvant tamoxifen: patient-level meta-analysis of randomised trials. Lancet. 2011;378:771-84.

14. Early Breast Cancer Trialists' Collaborative Group (EBCTCG), Peto R, Davies C, Godwin J, Gray R, Pan HC, et al. Comparisons between different polychemotherapy regimens for early breast cancer: meta-analyses of long-term outcome among 100,000 women in 123 randomised trials. Lancet. 2012;379: 432-44.

15. Kyndi M, Sorensen FB, Knudsen H, Overgaard M, Nielsen $\mathrm{HM}$, Overgaard J, et al. Estrogen receptor, progesterone receptor, HER-2, and response to postmastectomy radiotherapy in high-risk breast cancer: the Danish Breast Cancer Cooperative
Group. J Clin Oncol. 2008;26:1419-26.

16. Lowery AJ, Kell MR, Glynn RW, Kerin MJ, Sweeney KJ. Locoregional recurrence after breast cancer surgery: a systematic review by receptor phenotype. Breast Cancer Res Treat. 2012;133:831-41.

17. Sotiriou C, Neo SY, McShane LM, Korn EL, Long PM, Jazaeri A, et al. Breast cancer classification and prognosis based on gene expression profiles from a population-based study. Proc Natl Acad Sci U S A. 2003;100:10393-8.

18. Tseng YD, Uno H, Hughes ME, Niland JC, Wong YN, Theriault $\mathrm{R}$, et al. Biological subtype predicts risk of locoregional recurrence after mastectomy and impact of postmastectomy radiation in a large national database. Int J Radiat Oncol Biol Phys. 2015;93:622-30.

19. Voduc KD, Cheang MC, Tyldesley S, Gelmon K, Nielsen TO, Kennecke H. Breast cancer subtypes and the risk of local and regional relapse. J Clin Oncol. 2010;28:1684-91.

20. Jwa E, Shin KH, Lim HW, Jung SY, Lee S, Kang HS, et al. Identification of risk factors for locoregional recurrence in breast cancer patients with nodal stage N0 and N1: who could benefit from post-mastectomy radiotherapy? PLoS One. 2015;10: e0145463.

21. Kunkler IH, Canney P, van Tienhoven G, Russell NS; MRC/ EORTC (BIG 2-04) SUPREMO Trial Management Group. Elucidating the role of chest wall irradiation in 'intermediate-risk' breast cancer: the MRC/EORTC SUPREMO trial. Clin Oncol (R Coll Radiol). 2008;20:31-4. 